\title{
Lengths of Orthologous Prokaryotic Proteins Are Affected by Evolutionary Factors
}

\author{
Tatiana Tatarinova, ${ }^{1}$ Bilal Salih, ${ }^{2,3}$ Jennifer Dien Bard, ${ }^{1}$ \\ Irit Cohen, ${ }^{2,4}$ and Alexander Bolshoy ${ }^{2}$ \\ ${ }^{1}$ Children's Hospital Los Angeles, Keck School of Medicine, University of Southern California, \\ Los Angeles, CA 90027, USA \\ ${ }^{2}$ Department of Evolutionary and Environmental Biology and Institute of Evolution, University of Haifa, \\ 3498838 Haifa, Israel \\ ${ }^{3}$ Department of Computer Science, University of Haifa, 3498838 Haifa, Israel \\ ${ }^{4}$ The Tauber Bioinformatics Research Center, University of Haifa, 3498838 Haifa, Israel \\ Correspondence should be addressed to Tatiana Tatarinova; tatiana.tatarinova@usc.edu \\ and Alexander Bolshoy; bolshoy@research.haifa.ac.il
}

Received 8 September 2014; Accepted 2 November 2014

Academic Editor: Vassily Lyubetsky

Copyright (C) 2015 Tatiana Tatarinova et al. This is an open access article distributed under the Creative Commons Attribution License, which permits unrestricted use, distribution, and reproduction in any medium, provided the original work is properly cited.

\begin{abstract}
Proteins of the same functional family (for example, kinases) may have significantly different lengths. It is an open question whether such variation in length is random or it appears as a response to some unknown evolutionary driving factors. The main purpose of this paper is to demonstrate existence of factors affecting prokaryotic gene lengths. We believe that the ranking of genomes according to lengths of their genes, followed by the calculation of coefficients of association between genome rank and genome property, is a reasonable approach in revealing such evolutionary driving factors. As we demonstrated earlier, our chosen approach, Bubble-sort, combines stability, accuracy, and computational efficiency as compared to other ranking methods. Application of Bubble Sort to the set of 1390 prokaryotic genomes confirmed that genes of Archaeal species are generally shorter than Bacterial ones. We observed that gene lengths are affected by various factors: within each domain, different phyla have preferences for short or long genes; thermophiles tend to have shorter genes than the soil-dwellers; halophiles tend to have longer genes. We also found that species with overrepresentation of cytosines and guanines in the third position of the codon $\left(\mathrm{GC}_{3}\right.$ content) tend to have longer genes than species with low $\mathrm{GC}_{3}$ content.
\end{abstract}

\section{Introduction}

To better understand the interaction between the environment and bacteria, whether in a human host or any other ecosystem, one must know the laws governing prokaryotic evolution and adaptation to environment. For example, it is essential to study how a change in $\mathrm{pH}$ or external temperature affects a bacterial genome and especially its coding sequences. Unfortunately, the laws of prokaryotic coding sequence evolution remain unclear. Orthologous proteins may drastically differ in both codon usage and length across species. When a gene length changes, a protein may acquire a new function or lose an existing one, hence, changing the entire ecosystem. Many studies have analyzed the relationship between codon usage and the environment [1-3], but a few efforts were made to predict the effect of a changing environment on gene length. The main results were related to comparative analysis between protein lengths in eukaryotes and prokaryotes. Detailed comparison of protein length distributions in eukaryotes and prokaryotes can be found in $[4,5]$. Wang et al. [6] proposed that "molecular crowding" effect and evolution of linker sequences can explain differences between length of orthologous sequences in super-kingdoms. Our study is focused on protein lengths in prokaryotes, exclusively.

How does gene length change occur in prokaryotes? The main driving force in shaping gene length is a point mutation [7]. Point mutations may cause a stop codon shift, when the existing stop codon is destroyed and gene length is increased, a start codon drift, or appearance of a premature stop codon. To understand trends of fixation of mutations 
changing protein lengths we performed a comparative study of lengths of paralogs. We explore the use of seriation of genomes based on paralogs' lengths.

In recent papers $[8,9]$, we formulated the genome ranking problem, listed several approaches to solve it, described a novel method for genome ranking according to gene lengths, and demonstrated preliminary results from the ranking of prokaryotic genomes. These results indicated that hyperthermophilic species have shorter genes than mesophilic organisms. We hypothesize that gene lengths are not randomly distributed; instead they are affected by a number of environmental, genomic and taxonomic factors. In this paper we present a framework for analysis of gene lengths and evaluate effects of environmental factors.

In order to analyze evolutionary pressures acting on genes it is necessary to group them into well-defined functional categories. There are several existing approaches. First of all, there is the most popular database of Clusters of Orthologous Groups (COG) of proteins, which is a comprehensive collection of prokaryotic gene families. This database was created to classify the complete complement of proteins encoded by complete genomes based on evolutionary development. The data in COGs are updated continuously following the sequencing of new prokaryotic genomic sequences. As described by Tatusov et al. [10], the COGs database is a growing and useful resource to identify genes and groups of orthologs in different species that are related by evolution. Sixteen years ago, the database was started with only seven Bacterial genomes; in 2010 the database consisted of proteins from 52 Archaeal and 601 Bacterial genomes (a total of 653 complete genomes) that were assigned to 5,663 COGs; currently it contains approximately $2 \mathrm{~K}$ genomes.

The COG database is not the only possible data compilation to classify prokaryotic proteins. Since its publication over a decade ago, additional classifications have appeared. In 2007, Archaea were grouped into the acCOG database [11]. Another alternative, the eggNOG database $[12,13]$, grouped gene families at the universal level, covering all three domains of life.

Recently, Bolshoy et al. introduced a "gene-length based" model $[14,15]$, representing genomes as vectors of genes. The set of genomes is represented as a matrix, in which each row stands for a genome and each column stands for a gene family. Therefore, each element of this matrix stands for the length of a member of a gene family $i$ in a genome $j$. In our study, the objects are annotated prokaryotic genomes; the descriptors are the lengths of the genome proteins indexed according to the COG database.

A ranking is a relationship between a set of objects such that, for any two objects, the first is either ranked "higher than," "lower than," or "equal to" the second. Gene ranking is a useful approach to answer biological questions, however it is sometimes difficult to implement. Here we bring examples of usage this measure in biologic sciences. A prioritization or ranking is used in bioinformatics to aid in the discovery of disease-related genes. Computational methods are employed for ranking the genes according to their likelihood of being associated with the disease. A variety of methods have been conceived by the researchers for the prioritization of the disease candidate genes. A review of various aspects of computational disease gene prioritization and related problems is presented in Gill et al. [16].

In our case, the goal is to order the genomes that are represented as rows of a gene length matrix. There are different possible approaches to define the optimal rank of rows in the matrix. We have previously determined [9] that Bubble Sort method (B-Sort, see Section 5) is more accurate than Average Sort and Simple Additive Ranking and it is as accurate and significantly faster than the Simulated Annealing Procedure.

The complexity of the ranking problem using matrices with missing values was discussed in detail [17]. The same ranking problem appears in several areas of operations research, such as in the context of group decision making [18] and country-credit risk rating [19]. Missing data as well as variable relative importance of different gene families make the problem increasingly complex. To the best of our knowledge, genome ranking problem has been addressed for the first time in $[8,9]$.

Establishing ordered lists of genomes using lengths of coding sequences of orthologous genes, we aim to find an association between a genome rank and a genome property of interest, such as its role in virulence and adaptation. There are many different types of such properties: a prokaryote can be either Archaea or Bacteria; an organism may be hyperthermophile, thermophile, psychrophile, or mesophile; a genome has a certain GC-content, and so on. In summary, the goal is to find out whether gene lengths of a genome are associated with various genome properties and to measure the magnitude of this association. These findings will allow us to determine important factors such as virulence, biofilm formation, and antimicrobial resistance that may be associated with the pathogenesis of a specific species and the ability to cause serious infections in patients.

\section{Results}

We used a dataset of 1390 genomes (the "big" dataset) and a randomly selected subset of 100 genomes (the "small" dataset). For each dataset we used complete and filtered versions. The filtering procedure (see Section 5) removes those COGs that are present in only a small number of genomes and are likely to skew the ordering results. We set the frequency threshold to be $35 \%$, meaning that the filtering procedure removes COGs present in less than $35 \%$ of analyzed genomes. After filtering we obtained the filtered dataset containing 1474 COGs.

We assessed the consistency of ranks of genomes of the small dataset in two orderings: of the entire collection of 1390 genomes and of the subset of 100 genomes (Figure 1). We determined corresponding ranks of 100 genomes in the B-sorted dataset of 1390 genomes and discovered that the two orderings of 100 genomes were highly consistent (with correlation coefficient of 0.95 ). This confirms that the ranking procedure is stable. However, random selection of a small subset may cause wrong ranks of a few isolated genomes. Indeed, there are some genomes that show differences in 100 and 1390 genomes rank, for example, bacteria Sodalis glossinidius, which is ranked 42 in 100 genomes and 162 
TABLE 1: B-sort results (one run) for 1390 genomes, archaea.

\begin{tabular}{|c|c|c|c|c|c|}
\hline Phylum & Average rank & StDev & Median rank & Rank range & Number of genomes \\
\hline Crenarchaeota & 189 & 179 & 77 & $7-492$ & 35 \\
\hline Euryarchaeota & 312 & 297 & 233 & $5-1263$ & 74 \\
\hline Korarchaeota & 169 & NA & 169 & $169-169$ & 1 \\
\hline Nanoarchaeota & 5 & NA & 5 & $5-5$ & 1 \\
\hline Thaumarchaeota & 347 & 239 & 347 & $178-516$ & 2 \\
\hline Unclassified archaea & 771 & NA & 771 & $771-771$ & 1 \\
\hline
\end{tabular}

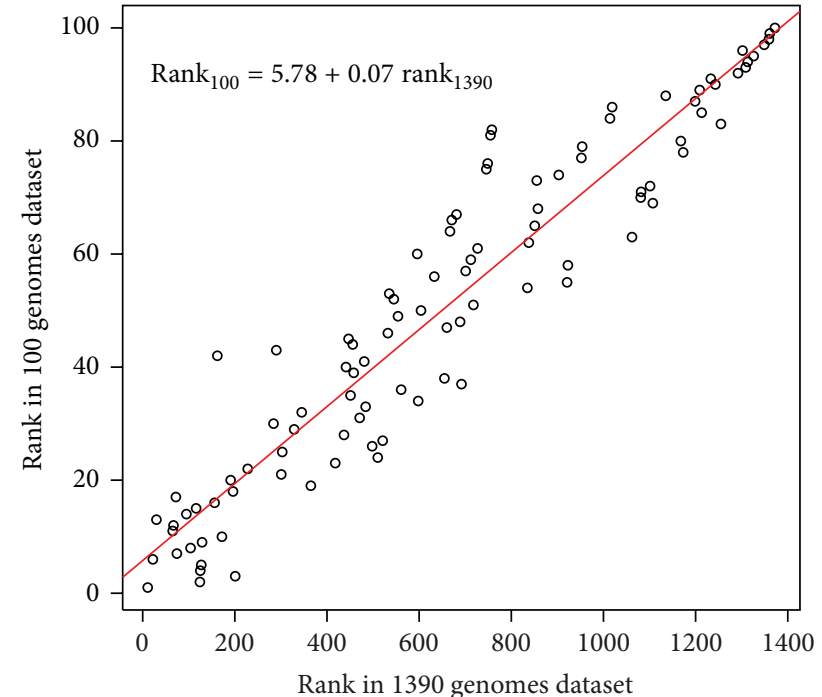

FIGURE 1: Consistency of Bubble Sort ranks in 1390 and 100 genomes datasets. Pearson's correlation coefficient between two ranks is 0.95 ; Kendall tau correlation coefficient is 0.82 .

in 1390 genomes dataset. Therefore, the ranks' consistency found for the huge majority of ranks is an additional support to the chosen method of ranking.

Let us start with an overview of the orderings; let us compare ranks of Bacteria and Archaea. (Larger value of a genome rank means longer genes in this genome.) Bacterial genomes have a broader distribution of ranks than Archaeal genomes (Figure 2). Overall, Bacterial ranks are larger than Archaeal ranks in the 1390 genome, as well as in 100 genome datasets. This observation can be illustrated using the violin plot of ranks' distributions, as shown in Figure 2. Average rank of 1276 Bacterial genomes was 735 and average rank of 114 Archaeal genomes was 254. This visual observation is also supported by a simple statistical procedure. Using the Wilcoxon rank test and $\alpha=0.01$, we calculated the test statistic $T_{a}$, equal to the sum of the ranks for the ordered data that belong to Archaea. $T_{a}$ was 28,913. For large samples $T_{a}$ is approximately normal with expected value and standard deviation calculated as

$$
\begin{gathered}
E\left(T_{a}\right)=\frac{n_{a}\left(n_{b}+n_{a}+1\right)}{2}=79,287, \\
\sigma\left(T_{a}\right)=\sqrt{\frac{n_{a} n_{b}\left(n_{a}+n_{b}+1\right)}{12}}=4106.3 .
\end{gathered}
$$

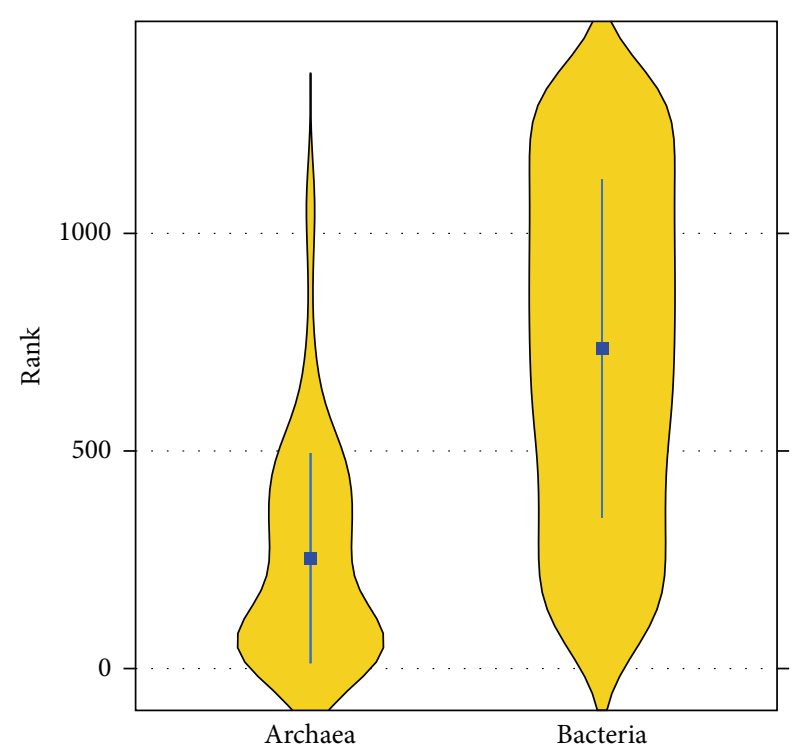

FIgURE 2: Violin plots of Bubble sort ranks of Archaea and Bacteria. Average rank of 1276 Bacterial genomes is 735 and average rank of 114 Archaeal genomes is 254.

Therefore,

$$
\begin{gathered}
Z=\frac{T_{a}-E\left(T_{a}\right)}{\sigma\left(T_{a}\right)}=-12.27, \\
P(Z<-12.27) \approx 10^{-34}<0.01 .
\end{gathered}
$$

Hence, we conclude that Bacterial genomes rank significantly higher than Archaeal genomes. Tables 1 and 2 show the summary statistics for the ordering of Archaeal and Bacterial genomes. These tables show mean, median, range, and standard deviation of Archaeal and Bacterial ranks of 1390 genomes stratified by phylum. In the Bacterial domain, Firmicutes and Thermotogae have shorter genes and Actinobacteria have longer ones. In the Archaeal domain, Euryarchaeota have longer genes than Crenarchaeota.. These results are consistent with our earlier findings from analysis of 100 prokaryotic genomes [8].

Next, we considered the nucleotide composition of coding regions. In prokaryotes, the nucleotide composition of coding regions varies significantly between species. $\mathrm{GC}_{3}$ (frequency of cytosine and guanine in the third position of the codon) is one of the variable features. Across the Bacterial domain, $\mathrm{GC}_{3}$ ranges from $10 \%$ to $90 \%$ [20]. Tatarinova et al. 
TABLE 2: B-sort results for 1390 genomes, bacteria.

\begin{tabular}{|c|c|c|c|c|c|}
\hline Phylum & Average rank & STD & Median rank & Rank range & Number of genomes \\
\hline Actinobacteria & 1223 & 166 & 1260 & $343-1390$ & 137 \\
\hline Aquificae & 182 & 79 & 168 & $82-306$ & 8 \\
\hline Bacteroidetes/Chlorobi & 992 & 188 & 1071 & $502-1359$ & 71 \\
\hline Candidatus Cloacamonas & 1054 & NA & 1054 & $1054-1054$ & 1 \\
\hline Chlamydiae/Verrucomicrobia & 1076 & 81 & 1079 & $835-1223$ & 25 \\
\hline Chloroflexi & 774 & 520 & 1109 & $70-1274$ & 15 \\
\hline Chrysiogenetes & 545 & NA & 545 & $545-545$ & 1 \\
\hline Cyanobacteria & 938 & 209 & 975 & $619-1276$ & 40 \\
\hline Deferribacteres & 205 & NA & 205 & $205-205$ & 1 \\
\hline Deinococcus-Thermus & 607 & 282 & 566 & $263-1126$ & 12 \\
\hline Dictyoglomi & 207 & 49 & 207 & $172-242$ & 2 \\
\hline Elusimicrobia & 412 & 143 & 412 & $311-513$ & 2 \\
\hline Fibrobacteres/Acidobacteria & 1171 & 172 & 1240 & $839-1293$ & 6 \\
\hline Firmicutes & 307 & 188 & 286 & $21-1387$ & 271 \\
\hline Fusobacteria & 462 & 100 & 461 & $361-564$ & 4 \\
\hline Gemmatimonadetes & 1214 & NA & 1214 & $1214-1214$ & 1 \\
\hline Nitrospirae & 563 & 418 & 563 & $267-858$ & 2 \\
\hline Planctomycetes & 1364 & 29 & 1368 & $1319-1389$ & 5 \\
\hline Proteobacteria & 759 & 325 & 775 & $1-1379$ & 588 \\
\hline Spirochaetes & 1050 & 155 & 1066 & $700-1317$ & 31 \\
\hline Synergistetes & 466 & 40 & 466 & $438-494$ & 2 \\
\hline Tenericutes & 657 & 223 & 631 & $92-1092$ & 36 \\
\hline Thermobaculum & 1049 & NA & 1049 & $1049-1049$ & 1 \\
\hline Thermodesulfobacteria & 458 & 32 & 458 & $435-480$ & 2 \\
\hline Thermotogae & 253 & 165 & 203 & $45-566$ & 12 \\
\hline
\end{tabular}

previously demonstrated $[21,22]$ that, within one eukaryotic species, $\mathrm{GC}_{3}$ content can be used to distinguish two classes (housekeeping and stress-specific) genes. Currently, we sought to evaluate mutation pressure acting on the entire prokaryotic genome by examining how the average $\mathrm{GC}_{3}$ content, calculated across all genes in a genome, is related to the position of the genome in a global ordering. We calculated the $\mathrm{GC}_{3}$ content of coding regions across all analyzed genomes and discovered that the genome rank and cytosine/guanine content of the third codon position of genes are positively correlated (Spearman rank correlation coefficient $\rho\left(\mathrm{GC}_{3}\right.$, rank $)=0.62$ for Bacteria and $\rho\left(\mathrm{GC}_{3}\right.$, rank $)=0.59$ for Archaea $)$. For example, the average $\mathrm{GC}_{3}$ content in Actinobacteria (0.70) is twice the amount seen in Firmicutes (0.35).

\section{Discussion}

The ability of some species to grow at high temperatures has been a long-term fascination of microbiologists. Proteins of hyperthermophilic species are more resilient to heat and are shorter than proteins of mesophilic species. Understanding this effect is important for biotechnology [23].

Up to now, less than a dozen studies were devoted to protein length distribution. Among those, there were only four relevant publications: $[4,5,8,24]$. In 2000, using an early version of the COG database, Zhang compared 22 species in three domains of life [4] and found that the average gene length is smallest for Archaea and greatest for eukaryotes. Similarly, Skovgaard et al. [24] analysed 34 prokaryotic genomes and discovered that, for the vast majority of functional families, Bacterial proteins were longer than Archaeal ones. In 2005, Brocchieri and Karlin [5] confirmed these findings using a larger collection of genomes (16 Archaeal and 67 Bacterial species). They found that bacteria were enriched in functional families with longer genes. In addition, they described a negative correlation between protein length and optimal growth temperature of Archaea and Bacteria. By grouping proteins into broad functional classes (information storage and processes; cellular processes; metabolism; poorly characterized; not characterized) and comparing their median lengths, Brocchieri and Karlin concluded that "information storage and processes" proteins are shorter than "cellular processes" and "metabolism" proteins. They also found that Archaea have more of the shorter and poorly characterized proteins.

The above mentioned studies, performed on relatively small sets of genomes, share the same deficiency of using average (mean or median) lengths of genes in a genome to reach their conclusions. As we illustrated in $[8,9]$ this approach can substantially distort results. In $[8,9]$ we proposed a systematic framework to analyse the relationship of prokaryotic gene lengths and environmental conditions that is not based on analysis of average lengths of proteins. This 
framework, further investigated in the current paper, allows more flexibility and produces more meaningful results than the previous approaches.

Hyperthermophilic species of Archaea and Bacteria, living in extreme environments (such as volcanic hot springs) occupy the top portions of the ranking lists of the small and big datasets. At a first glance it appears that we could hypothesize that extremophiles have shorter genes than species living under normal conditions. However, the situation appears to be more complex. For illustration we consider Sulfolobales, Thermoproteales, and Halobacteriales. Sulfolobales grow in volcanic hot springs at $\mathrm{pH} 2-3$ and a temperature of 7580 degrees Celsius. In the ordered list of 1390 genomes, Sulfolobales occupy positions from 12 to 94 , which means that as a rule Sulfolobales have very short genes. Thermoproteales (extremely thermoacidophilic anaerobic Archaea isolated from Icelandic solfataras) also have very short genes, their genomes are found in positions from 7 to 77 but also in positions from 412 to 460 in the ordered list, which are positions of genomes with moderately short genes. Halobacteriales (found in water saturated or nearly saturated with salt) are placed in positions from 541 to 1263 which are not considered genomes with short genes. From these observations follows that stress of living in an arbitrary extreme environment is not the factor, while, probably, hyperthermophilicity and halophilicity are the factors affecting orderings in opposite directions.

We also showed that, as a group, Bacterial genomes are ranked significantly higher than the Archaeal ones according to the length of their genes (Figure 2). This observation may be explained by the fact that the vast majority of completely sequenced Archaeal genomes are hyperthermophiles, which tend to have shorter genes as compared to psychrophiles and mesophiles. Our previous speculations obtained on relatively small datasets [8] and our current results on 1390 genome dataset are consistent with the hypothesis that high temperature environment is a factor causing reduction of gene length. In the 100-genome dataset hyperthermophiles occupy positions in the top portion of the list: top 20 in the 100 genomes list. They are also ranked in the top of the 1390 genome dataset.

We also observed that $34 \%$ of the shortest (first 100 positions in the ordered list) of 1390 genomes are occupied by hyperthermophilic species, while none are found in the longest (last 100 in the ordered list). Furthermore, 90\% of thermophiles are placed in the top third of the list. Moderately thermophilic species are not restricted to the top positions. For example, Thermobifida fusca (a moderately thermophilic soil bacterium growing at $55^{\circ} \mathrm{C}$ and a major degrader of plant cell walls in heated organic materials such as compost heaps, rotting hay, manure piles or mushroom growth medium) occupies position 1260 in the ordered list. Anaerolinea thermophila, with similar growth temperature, has a close position of 1173 .

There are several remarkable features that appeared as a result of the 1390 genome ordering. Campylobacterales (belonging to the phylum Proteobacteria) have an average position of 203, with the smallest position of 10 (Helicobacter bizzozeronii ciii-1) and the largest position of 392 (Helicobacter hepaticus atcc 51449). Most species in this family are human and animal pathogens. Namely, Campylobacter jejuni is a microaerophilic bacterium frequently associated with gastroenteritis in humans. Complications such as meningitis [25], septicemia [26], and Guillain-Barré syndrome have also been reported [27]. In addition, Helicobacter bizzozeronii (position 10) has been implicated in gastric infections, similar to Helicobacter pylori, referred to as non-Helicobacter pylori Helicobacter (NHPH) infections in humans [28]. It appears that all known Campylobacterales have short genomes. It is tempting to speculate that there are evolutionary pressures to keep genes in short pathogenic genomes as short as possible.

However, not all pathogens have short genes. Not even all pathogens with short genomes have short genes. Common obligate intracellular prokaryotic pathogens from the phylum of Chlamydiae are very small (measuring $0.3-0.6 \mu \mathrm{m}$ in diameter) and grow by infecting eukaryotic host cells. This phylum is comprised of several major intracellular pathogens of humans and animals, causing a variety of diseases. These bacteria can cause keratoconjunctivitis, pneumonitis, and sexually transmitted infections. In spite of its small physical dimensions, Chlamydiae have exceptionally long genes: the ranks of 21 members of this class are located from positions 835 to 1127 in the ranking list. We speculate that there are certain evolutionary factors (yet to be discovered) that keep Chlamydiae genes so long.

As we see, Campylobacterales (of the phylum Proteobacteria), have short genes. At the opposite end of the length spectrum we find the phylum Actinobacteria, tending to have longer genes. Only 8 out of 137 species of Actinobacteria have positions below 1000 in the ordered set. One of the species, a pathogenic bacterium Renibacterium salmoninarum [29], was placed among species with characteristically short genes in the position 343. The genome of $R$. salmoninarum has extended regions of synteny to the Arthrobacter sp. strain FB24 and Arthrobacter aurescens TC1 genomes, but it is approximately $1.9 \mathrm{Mb}$ smaller than two sequenced Arthrobacter genomes and has a lower GC content [29]. In the Bubble Sort list, Arthrobacters occupy positions 1230, 1301, 1342, 1343, and 1354. Our results show that significant genome reduction, which has occurred since divergence from the last common ancestor, affected not only gene content but also lengths of remaining genes. It is possible that factors affecting gene lengths of Actinobacteria are different from the factors acting on Chlamydiae, while resulting in keeping proteins longer in both cases.

Relationships between gene length and codon bias have been previously studied by [30-33]. Oliver and Marín [30] and Xia et al. [32] observed a positive correlation between length and GC composition of coding sequences in prokaryotes, attributing the effect to reduced frequency of stop codons in GC-rich species. Later Xia et al. [33] mentioned that the correlation is weak for a number of species, with 4 species showing a negative correlation. Thus Xia et al. formulated a more general hypothesis incorporating selection against cytosine (C) usage. In [33] they described two additional factors giving rise to this selection: transcription efficiency and "insurance" against cytosine deamination. 
Third positions in codon are largely degenerate; 70\% of changes at third codon positions are synonymous [34]. Therefore, it makes sense to analyze adaptation effects using GC composition in the third position of the codon, $\mathrm{GC}_{3}$. We showed that adaptation to higher temperatures affects the genome in two ways: first, $\mathrm{GC}_{3}$ content of genes tends to increase with growth temperature [35]; at the same time, hyperthermophilic species tend to have shorter genes as it can be seen from the ranks of these species both in the 100genome dataset and in the larger dataset. Several factors may compete for placement of the Bacterial species in the ordering rank. Adaptation to high temperatures and pathogenicity may tend to place an organism into lower ranks. High $\mathrm{GC}_{3}$ composition and adaptation to high salinity environments places an organism into higher ranks. However, future research is needed to determine important factors, both environmental and genomic, that may affect the rank of the genome. This information will allow us to further understand and possibly predict the invasive or virulent nature of a particular species compared to a nonpathogenic organism that is part of the normal commensal flora of an individual. Further exploration of these factors may also answer questions on the emerging mechanisms of resistance that may be associated with specific organisms and on prediction of resistance using novel methods other than conventional susceptibility tests.

We will continue updating our collection of prokaryotic genome orderings. When a new genome is sequenced, it is not necessary to repeat the entire ranking procedure from an unordered dataset. In order to incorporate a newly sequenced genome in our analysis, it is necessary to (1) predict genes and (2) assign COG categories. Then the new, completely annotated, genome can be added to the presorted data matrix, using average gene length as a rough indicator of the new genome position. Then the ranking procedure should be applied to the updated matrix. Since all but one of the genomes is already in the correct place, the ranking procedure will have to make only a small number of steps to determine the rank of a new genome.

\section{Conclusions}

We applied Bubble Sort to the set of 1390 prokaryotic genomes and revealed several interesting trends. We demonstrated that hyperthermophiles may be always characterized as having short proteins. Also, the resulting ordering showed that Archaea have shorter genes than Bacteria, and we speculate that this can be attributed to the prevalence of hyperthermophiles among the sequenced Archaea. Within each domain, different phyla have preferences for short or long genes. Another interesting observation is the significant correlation between gene length and GC composition of coding regions. Therefore, we suggest that gene lengths are not randomly distributed across species but are shaped by environmental and genomic factors.

The genome ranking procedure is stable. Inclusion of additional genomes does not distort the relative ranking of genomes. The correlation coefficient between the ranks of the 100 genomes in the 100-genome dataset and in the larger (1390) dataset is 0.95. Hyperthermophilic species are ranked on top in both 100 and 1390-genome lists; soil dwelling species are consistently at the bottom of the list.

Our results show that environmental factors constitute a strong force that groups evolutionary distant species together in protein-lengths' ranking. On the other hand, evolutionary history and phylogenetic closeness group certain organisms together as well. Relative influence of these factors varies between organisms. For example, we demonstrated that hyperthermophilic species have shorter genes than mesophilic organisms, which implies that environmental factors may affect gene length. However, not every environmental stress has the gene shortening effect. For example, high salinity represents an extreme environment that relatively few organisms have been able to adapt to and occupy. Halophiles are a type of extremophile organisms that live in high salt concentrations. Seemingly, high salinity opposite to high temperature does not cause protein-length decrease; the extreme halophiles (or halobacteria), tend to have pretty long genes.

\section{Materials and Methods}

All four ranking algorithms discussed in this paper were applied to input matrices based on the database of Clusters of Orthologous Groups of proteins (COG) [10, 36-38]. As of October 2012, there were 5664 COGs, 1276 Bacterial and 114 Archaeal genomes sequences in the NCBI database. The sequences were processed according to the procedures described below.

5.1. COGs Database. Information about every completely sequenced and annotated prokaryotic genome is stored as tables of protein features, called PTT files, prepared by the National Center for Biotechnology Information (NCBI). The complete collection of current PTT files can be found at $\mathrm{ftp}$ //ftp.ncbi.nih.gov/genomes/.

From every prokaryotic NCBI PTT file, we extracted information about each gene length, COG and added the genome index (tax id). We created a combined gene-length matrix, where rows correspond to genomes, identified by taxonomy id, and columns correspond to COGs. Each element $(i, j)$ of this matrix is a length of gene belonging to COG $i$ in genome $j$. All currently available genomes were described in these two files. To check the ranking methods described below we used small subsets (100 genomes) of this dataset.

5.2. Preprocessing Procedures. To get an input file for further ranking the following preprocessing procedures developed by Bolshoy et al. $[9,15,39]$ were applied.

(1) Selection of Genome Subsets. A subset may be defined applying different criteria: it may be either a representative sample, a taxaspecific subset, or randomly chosen genomes.

(2) Application of a Filtering Parameter (An Entry Threshold) on a Selected Subset. Only COGs containing more than a threshold number of genomes are considered for further processing. For example, if the filtering value is equal to $20 \%$ and an amount of genomes in 
TABle 3: List of Archaeal (A) and Bacterial (B) genomes in the Bubble Sort ordering rank, 100 genomes dataset. Hyperthermophiles, Streptococci, and Enterococci are marked in the Note column.

\begin{tabular}{|c|c|c|c|}
\hline Rank & Domain & Note & Organism \\
\hline 1 & $\mathrm{~A}$ & Hyperthermophile & Archaeoglobus fulgidus dsm 4304 \\
\hline 2 & A & Hyperthermophile & Thermoplasma volcanium gss1 \\
\hline 3 & $\mathrm{~B}$ & Hyperthermophile & Thermotoga sp. rq2 \\
\hline 4 & A & Hyperthermophile & Thermoplasma acidophilum dsm 1728 \\
\hline 5 & $\mathrm{~B}$ & Hyperthermophile & Thermotoga neapolitana dsm 4359 \\
\hline 6 & A & Hyperthermophile & Thermococcus onnurineus nal \\
\hline 7 & $\mathrm{~B}$ & & Campylobacter concisus 13826 \\
\hline 8 & $\mathrm{~B}$ & & Campylobacter curvus 525.92 \\
\hline 9 & $\mathrm{~B}$ & Hyperthermophile & Aquifex aeolicus vf5 \\
\hline 10 & $\mathrm{~B}$ & Hyperthermophile & Dictyoglomus thermophilum h-6-12 \\
\hline 11 & $\mathrm{~B}$ & & Bacillus cereus atcc 14579 \\
\hline 12 & $\mathrm{~B}$ & & Bacillus cytotoxicus nvh 391-98 \\
\hline 13 & $\mathrm{~B}$ & & Melissococcus plutonius atcc 35311 \\
\hline 14 & A & Hyperthermophile & Thermococcus sibiricus mm 739 \\
\hline 15 & $\mathrm{~B}$ & & Listeria monocytogenes clip81459 \\
\hline 16 & $\mathrm{~B}$ & & Bacillus amyloliquefaciens dsm 7 \\
\hline 17 & $\mathrm{~B}$ & & Rickettsia canadensis str. Mckiel \\
\hline 18 & A & Hyperthermophile & Pyrococcus abyssi ge5 \\
\hline 19 & $\mathrm{~B}$ & & Helicobacter felis atcc 49179 \\
\hline 20 & A & Hyperthermophile & Pyrococcus horikoshii ot 3 \\
\hline 21 & $\mathrm{~B}$ & Streptococcus & Streptococcus pneumoniae p1031 \\
\hline 22 & $\mathrm{~B}$ & Streptococcus & Streptococcus agalactiae a 909 \\
\hline 23 & $\mathrm{~B}$ & & Caldicellulosiruptor bescii dsm 6725 \\
\hline 24 & $\mathrm{~B}$ & & Mycoplasma fermentans $m 64$ \\
\hline 25 & $\mathrm{~B}$ & Streptococcus & Streptococcus agalactiae $2603 \mathrm{v} / \mathrm{r}$ \\
\hline 26 & A & & Methanosalsum zhilinae dsm 4017 \\
\hline 27 & $\mathrm{~B}$ & & Francisella sp. tx077308 \\
\hline 28 & $\mathrm{~B}$ & Streptococcus & Streptococcus equi subsp. zooepidemicus \\
\hline 29 & $\mathrm{~B}$ & & Bacillus pumilus safr-032 \\
\hline 30 & $\mathrm{~B}$ & & Pediococcus pentosaceus atcc 25745 \\
\hline 31 & $\mathrm{~B}$ & & Geobacter lovleyi sz \\
\hline 32 & $\mathrm{~B}$ & Enterococcus & Enterococcus faecalis $v 583$ \\
\hline 33 & $\mathrm{~B}$ & & Natranaerobius thermophilus jw/nm-wn-lf \\
\hline 34 & $\mathrm{~B}$ & & Mycoplasma pulmonis uab ctip \\
\hline 35 & $\mathrm{~B}$ & & Brevibacillus brevis nbrc 100599 \\
\hline 36 & $\mathrm{~B}$ & & Mycoplasma genitalium g37 \\
\hline 37 & $\mathrm{~B}$ & & Mycoplasma leachii pg50 \\
\hline 38 & B & & Ureaplasma parvum serovar 3 \\
\hline 39 & $\mathrm{~B}$ & & Bacillus thuringiensis str. al hakam \\
\hline 40 & B & & Neisseria meningitidis 053442 \\
\hline 41 & $\mathrm{~B}$ & & Legionella pneumophila str. paris \\
\hline 42 & $\mathrm{~B}$ & & Sodalis glossinidius str. "morsitans" \\
\hline 43 & $\mathrm{~B}$ & & Candidatus riesia pediculicola usda \\
\hline 44 & B & & Lactobacillus gasseri atcc 33323 \\
\hline 45 & B & & Coxiella burnetii rsa 331 \\
\hline 46 & $\mathrm{~B}$ & & Laribacter hongkongensis hlhk9 \\
\hline 47 & B & & Ruminococcus albus 7 \\
\hline 48 & B & & Mycoplasma pneumoniae m129 \\
\hline 49 & A & & Halalkalicoccus jeotgali b3 \\
\hline
\end{tabular}


TABle 3: Continued.

\begin{tabular}{|c|c|c|c|}
\hline Rank & Domain & Note & Organism \\
\hline 50 & $\mathrm{~B}$ & & Geobacter uraniireducens rf4 \\
\hline 51 & B & & Brachyspira pilosicoli 95/1000 \\
\hline 52 & B & & Pseudogulbenkiania sp. nh8b \\
\hline 53 & B & & Dechloromonas aromatica $r c b$ \\
\hline 54 & B & & Maribacter sp. htcc 2170 \\
\hline 55 & B & & Zobellia galactanivorans \\
\hline 56 & B & & Escherichia coli bw2952 \\
\hline 57 & B & & Erwinia amylovora atcc 49946 \\
\hline 58 & B & & Gramella forsetii kt0803 \\
\hline 59 & B & & Klebsiella variicola at- 22 \\
\hline 60 & B & & Salmonella enterica subsp. arizonae serovar \\
\hline 61 & B & & Yersinia enterocolitica subsp. enterocolitica 8081 \\
\hline 62 & B & & Methylomonas methanica mc09 \\
\hline 63 & B & & Borrelia turicatae 91 e135 \\
\hline 64 & B & & Cronobacter turicensis z 3032 \\
\hline 65 & B & & Yersinia pseudotuberculosis pbl/+ \\
\hline 66 & B & & Xanthomonas oryzae pv. oryzae maff 311018 \\
\hline 67 & B & & Tropheryma whipplei tw08/27 \\
\hline 68 & B & & Spirochaeta smaragdinae dsm 11293 \\
\hline 69 & B & & Sphingobacterium sp. 21 \\
\hline 70 & B & & Dyadobacter fermentans dsm 18053 \\
\hline 71 & $\mathrm{~B}$ & & Eubacterium eligens atcc 27750 \\
\hline 72 & B & & Chlamydophila pneumoniae ar39 \\
\hline 73 & B & & Pelodictyon phaeoclathratiforme bu-1 \\
\hline 74 & B & & Desulfovibrio vulgaris str. hildenborough \\
\hline 75 & B & & Prosthecochloris aestuarii dsm 271 \\
\hline 76 & B & & Dinoroseobacter shibae dfl 12 \\
\hline 77 & B & & Acidiphilium cryptum jf-5 \\
\hline 78 & B & & Anaerolinea thermophila uni-1 \\
\hline 79 & B & & Thauera sp. mzlt \\
\hline 80 & B & & Magnetococcus sp. mc-1 \\
\hline 81 & B & & Sinorhizobium meliloti 1021 \\
\hline 82 & $\mathrm{~B}$ & & Bordetella petrii dsm 12804 \\
\hline 83 & B & & Chloroflexus aggregans dsm 9485 \\
\hline 84 & B & & Corynebacterium glutamicum $r$ \\
\hline 85 & B & & Cyanothece sp. pcc 7822 \\
\hline 86 & $\mathrm{~B}$ & & Starkeya novella dsm 506 \\
\hline 87 & B & & Arcanobacterium haemolyticum dsm 20595 \\
\hline 88 & B & & Rhodopseudomonas palustris $d x-1$ \\
\hline 89 & $\mathrm{~B}$ & & Rhodospirillum centenum sw \\
\hline 90 & B & & Xanthobacter autotrophicus py2 \\
\hline 91 & $\mathrm{~B}$ & & Mycobacterium leprae br4923 \\
\hline 92 & B & & Gluconacetobacter diazotrophicus pal 5 \\
\hline 93 & B & & Streptomyces griseus subsp. griseus nbrc 13350 \\
\hline 94 & B & & Streptomyces scabiei 87.22 \\
\hline 95 & B & & Intrasporangium calvum dsm 43043 \\
\hline 96 & B & & Burkholderia rhizoxinica hki 454 \\
\hline 97 & B & & Haliangium ochraceum dsm 14365 \\
\hline 98 & $\mathrm{~B}$ & & Salinibacter ruber $m 8$ \\
\hline 99 & B & & Rothia dentocariosa atcc 17931 \\
\hline 100 & $\mathrm{~B}$ & & Bifidobacterium animalis subsp. lactis ad011 \\
\hline
\end{tabular}


a subset is equal to 500, then only COGs containing at least 100 genomes are considered (passed the entry threshold).

(3) Sampling. If there are multiple instances of a COG related to the same genome, a median length value for all paralogs from the same genome and from the same COG is used for further processing.

5.3. Sets of Genomes. As of May 2012, there were approximately $1500 \mathrm{NC}$-numbers, corresponding to 1390 annotated prokaryotic genomes at NCBI. Multiple NC numbers occur for prokaryotes with more than one chromosome, such as Burkholderia cepacia (Tax id 269483). This large set was used for the final Bubble sort analysis. In that set, 114 genomes are Archaeal and 1276 are Bacterial. To compare performance of the methods, we used a small subset of this dataset, same as we used previously [8]. Then, we had randomly selected 100 prokaryotic genomes out of a possible 1390, contained at the NCBI COG database. This small set contains 9 Archaeal and 91 Bacterial genomes. The list of selected genomes is shown in Table 3. After the selection of genomes, we discarded those COGs that were present in less than $35 \%$ of those selected genomes. Upon filtering, our input contained 1455 COGs. Note, that the input file is a sparse matrix.

5.4. Bubble Sort Ranking (B-Sort). As a LOPI strategy [40] we apply here the regular "bubble sort" procedure [41] interchanging the rows of a given matrix. (In a simulation study on graphs [42], the LOPI strategies found a global maximum of the goal function defined on edges in the majority of the cases.) The criterion by which the procedure decides whether rows would be interchanged is as follows. Comparing two genomes we take into account only those COGs that both genomes have members in them. Comparing pairs of lengths of genes from relevant COGs we count which genome in a pair has longer genes more frequently. In other words, if a genome associated with a row $i$ has longer genes than has a genome associated with a row $i+1$, then these rows would be interchanged. We note that due to application to a sparse matrix this procedure would not necessarily lead to the optimal ordering.

5.5. Solving of the Optimization Problem. The three methods above are pretty intuitive. They do not have a goal to find an optimal ranking but the results have a good chance to be close to the optimal ranking. In our review [8] we described several procedures to find a nearly optimal ranking using approach from the field of combinatorial optimization. Maximization of an average Kendall tau rank correlation coefficient is one of them. As we presented it, the goal is to assign each genome $i$ to a scale $x$ such that $x_{i}$ most accurately recovers the across-genome gene lengths. "Most accurately" here means achieving the maximum of the function $x^{\tau}$ :

$$
x^{\tau}=\max _{x}\left[\sum_{k=1}^{K} \sum_{i=1}^{N-1} \sum_{j=i+1}^{N} C_{i j}\left(\vec{x}, \overrightarrow{r^{k}}\right)\right] \text {, }
$$

where given a rating vector $\vec{x}$ and an "individual" vector $\overrightarrow{r^{k}}$ of the gene lengths of COG $k, C_{i j}\left(\vec{x}, \overrightarrow{r^{k}}\right)$ is equal to 1 , if $\left(r_{x_{i}}^{k}<r_{x_{j}}^{k}\right)$, equal to $1 / 2$, if $\left(r_{x_{i}}^{k}=r_{x_{j}}^{k}\right)$, and 0 -otherwise.

5.6. Kemeny-Optimal Ranking. Kemeny-Optimal Ranking is an optimal rank aggregation approach. In $[43,44]$ the authors proposed a precise criterion for determining the "best" aggregate ranking. Given $n$ objects and $k$ permutations of the objects, $\left\{\pi_{1}, \pi_{2}, \ldots, \pi_{k}\right\}$, a Kemeny optimal ranking of the objects is the ranking $\pi$ that minimizes a "sum of distances" $P=\sum_{i=1}^{k} d\left(\vec{x}, \overrightarrow{r^{k}}\right)$, where $d\left(\vec{x}, \overrightarrow{r^{k}}\right)$, denotes a distance between a rating vector $\vec{x}$ and an "individual" vector $\overrightarrow{r^{k}}$ based on Kendall's $\boldsymbol{\tau}$ rank-correlation. From the properties of Kendall's $\tau$ rank-correlation it follows that a Kemeny optimal ranking minimizes the number of pairwise disagreements with the given $k$ rankings $x^{\tau}$ and maximizes sortedness.

It is known that finding a Kemeny optimal ranking is NPhard [45] and remains NP-hard even when there are only four input lists to aggregate [46]. This motivates the problem of finding a ranking that approximately minimizes the number of disagreements with the given input rankings.

\section{Conflict of Interests}

The authors declare that there is not conflict of interests regarding the publication of this paper.

\section{Authors' Contribution}

Tatiana Tatarinova curated the datasets, implemented the algorithm, carried out the comparison of algorithms, and wrote the paper. Bilal Salih and Irit Cohen implemented the algorithms and participated in writing of the Materials and Methods section. Jennifer Dien Bard provided clinical insight and participated in paper preparation. Alexander Bolshoy led the project, designed the framework, and wrote the paper. All authors read and approved the final paper.

\section{Acknowledgments}

Tatiana Tatarinova was supported by NIH: GM068968 and NIH-NICHD: HD070996. The authors would like to thank Professor Roger Jelliffe, USC, for proofreading the paper.

\section{References}

[1] H. Willenbrock, C. Friis, A. S. Juncker, and D. W. Ussery, "An environmental signature for 323 microbial genomes based on codon adaptation indices," Genome Biology, vol. 7, no. 12, article R114, 2006.

[2] M. Botzman and H. Margalit, "Variation in global codon usage bias among prokaryotic organisms is associated with their lifestyles," Genome Biology, vol. 12, no. 10, article R109, 2011.

[3] M. Roller, V. Lucić, I. Nagy, T. Perica, and K. Vlahoviček, "Environmental shaping of codon usage and functional adaptation 
across microbial communities," Nucleic Acids Research, vol. 41, no. 19, pp. 8842-8852, 2013.

[4] J. Zhang, "Protein-length distributions for the three domains of life," Trends in Genetics, vol. 16, no. 3, pp. 107-109, 2000.

[5] L. Brocchieri and S. Karlin, "Protein length in eukaryotic and prokaryotic proteomes," Nucleic Acids Research, vol. 33, no. 10, pp. 3390-3400, 2005.

[6] M. Wang, C. G. Kurland, and G. Caetano-Anollés, "Reductive evolution of proteomes and protein structures," Proceedings of the National Academy of Sciences of the United States of America, vol. 108, no. 29, pp. 11954-11958, 2011.

[7] A. A. Vakhrusheva, M. D. Kazanov, A. A. Mironov, and G. A. Bazykin, "Evolution of prokaryotic genes by shift of stop codons," Journal of Molecular Evolution, vol. 72, no. 2, pp. 138146, 2011.

[8] A. Bolshoy and T. Tatarinova, "Methods of combinatorial optimization to reveal factors affecting gene length," Bioinformatics and Biology Insights, vol. 6, pp. 317-327, 2012.

[9] A. Bolshoy, B. Salih, I. Cohen, and T. Tatarinova, "Ranking of prokaryotic genomes based on maximization of sortedness of gene lengths," Journal of Data Mining in Genomics \& Proteomics, vol. 5, article 151, 2014.

[10] R. L. Tatusov, E. V. Koonin, and D. J. Lipman, "A genomic perspective on protein families," Science, vol. 278, no. 5338, pp. 631-637, 1997.

[11] Y. I. Wolf, K. S. Makarova, N. Yutin, and E. V. Koonin, “Updated clusters of orthologous genes for Archaea: a complex ancestor of the Archaea and the byways of horizontal gene transfer," Biology Direct, vol. 7, article 46, 2012.

[12] L. J. Jensen, P. Julien, M. Kuhn et al., "eggNOG: automated construction and annotation of orthologous groups of genes," Nucleic Acids Research, vol. 36, no. 1, pp. D250-D254, 2008.

[13] S. Powell, D. Szklarczyk, K. Trachana et al., "eggNOG v3.0: Orthologous groups covering 1133 organisms at 41 different taxonomic ranges," Nucleic Acids Research, vol. 40, no. 1, pp. D284-D289, 2012.

[14] A. Bolshoy and Z. Volkovich, "Whole-genome prokaryotic clustering based on gene lengths," Discrete Applied Mathematics, vol. 157, no. 10, pp. 2370-2377, 2009.

[15] A. Bolshoy, Z. Volkovich, V. Kirzhner, and Z. Barzily, Genome Clustering: From Linguistic Models to Classification of Genetic Texts, Springer, Berlin, Germany, 2010.

[16] N. Gill, S. Singh, and T. C. Aseri, "Computational disease gene prioritization: an appraisal," Journal of Computational Biology, vol. 21, no. 6, pp. 456-465, 2014.

[17] D. S. Hochbaum, E. Moreno-Centeno, P. Yelland, and R. A. Catena, "Rating customers according to their promptness to adopt new products," Operations Research, vol. 59, no. 5, pp. 1171-1183, 2011.

[18] D. S. Hochbaum and A. Levin, "Methodologies and algorithms for group-rankings decision," Management Science, vol. 52, no. 9, pp. 1394-1408, 2006.

[19] D. S. Hochbaum and E. Moreno-Centeno, "Country creditrisk rating aggregation via the separation-deviation model," Optimization Methods and Software, vol. 23, no. 5, pp. 741-762, 2008.

[20] A. Muto and S. Osawa, "The guanine and cytosine content of genomic DNA and bacterial evolution," Proceedings of the National Academy of Sciences of the United States of America, vol. 84, no. 1, pp. 166-169, 1987.
[21] T. V. Tatarinova, N. N. Alexandrov, J. B. Bouck, and K. A. Feldmann, "GC3 biology in corn, rice, sorghum and other grasses," BMC Genomics, vol. 11, no. 1, article 308, 2010.

[22] T. Tatarinova, E. Elhaik, and M. Pellegrini, "Cross-species analysis of genic $\mathrm{GC}_{3}$ content and DNA methylation patterns," Genome Biology and Evolution, vol. 5, no. 8, pp. 1443-1456, 2013.

[23] I. Lasa and J. Berenguer, "Thermophilic enzymes and their biotechnological potential," Microbiologia, vol. 9, no. 2, pp. 7789, 1993.

[24] M. Skovgaard, L. J. Jensen, S. Brunak, D. Ussery, and A. Krogh, "On the total number of genes and their length distribution in complete microbial genomes," Trends in Genetics, vol. 17, no. 8, pp. 425-428, 2001.

[25] K. Tsoni, E. Papadopoulou, E. Michailidou, and I. Kavaliotis, "Campylobacter jejuni meningitis in a neonate: a rare case report," Journal of Neonatal-Perinatal Medicine, vol. 6, no. 2, pp. 183-185, 2013.

[26] H. Nadorlik, M. Marcon, K. Koranyi, O. Ramilo, and A. Mejias, "A 2-month-old with bacteremia and gastroenteritis," Pediatric Infectious Disease Journal, vol. 31, no. 2, pp. 210-216, 2012.

[27] M. Zhang, L. He, Q. Li et al., "Genomic characterization of the Guillain-Barre syndrome-associated Campylobacter jejuni ICDCCJ07001 isolate," PLoS ONE, vol. 5, no. 11, Article ID e15060, 2010.

[28] B. Flahou, F. Haesebrouck, A. Smet, H. Yonezawa, T. Osaki, and S. Kamiya, "Gastric and enterohepatic non-Helicobacter pylori Helicobacters," Helicobacter, vol. 18, supplement 1, pp. 66-72, 2013.

[29] G. D. Wiens, D. D. Rockey, Z. Wu et al., "Genome sequence of the fish pathogen Renibacterium salmoninarum suggests reductive evolution away from an environmental arthrobacter ancestor," Journal of Bacteriology, vol. 190, no. 21, pp. 6970-6982, 2008.

[30] J. L. Oliver and A. Marín, "A relationship between GC content and coding-sequence length," Journal of Molecular Evolution, vol. 43, no. 3, pp. 216-223, 1996.

[31] E. N. Moriyama and J. R. Powell, "Gene length and codon usage bias in Drosophila melanogaster, Saccharomyces cerevisiae and Escherichia coli," Nucleic Acids Research, vol. 26, no. 13, pp. 31883193, 1998.

[32] X. Xia, Z. Xie, and W.-H. Li, "Effects of GC content and mutational pressure on the lengths of exons and coding sequences," Journal of Molecular Evolution, vol. 56, no. 3, pp. 362-370, 2003.

[33] X. Xia, H. Wang, Z. Xie, M. Carullo, H. Huang, and D. Hickey, "Cytosine usage modulates the correlation between CDS length and CG content in prokaryotic genomes," Molecular Biology and Evolution, vol. 23, no. 7, pp. 1450-1454, 2006.

[34] E. Elhaik and T. Tatarinova, "GC3 biology in eukaryotes and prokaryotes," in DNA Methylation-From Genomics to Technology, T. Tatarinova and O. Kerton, Eds., 2012, http://www intechopen.com/books/dna-methylation-from-genomics-totechnology/gc3-biology-in-eukaryotes-and-prokaryotes.

[35] S. Basak, T. Banerjee, S. K. Gupta, and T. C. Ghosh, "Investigation on the causes of codon and amino acid usages variation between thermophilic Aquifex aeolicus and mesophilic Bacillus subtilis," Journal of Biomolecular Structure and Dynamics, vol. 22, no. 2, pp. 205-214, 2004.

[36] R. L. Tatusov, N. D. Fedorova, J. D. Jackson et al., "The COG database: an updated version includes eukaryotes," BMC Bioinformatics, vol. 4, article 41, 2003. 
[37] R. L. Tatusov, M. Y. Galperin, D. A. Natale, and E. V. Koonin, "The COG database: a tool for genome-scale analysis of protein functions and evolution," Nucleic Acids Research, vol. 28, no. 1, pp. 33-36, 2000.

[38] R. L. Tatusov, D. A. Natale, I. V. Garkavtsev et al., "The COG database: New developments in phylogenetic classification of proteins from complete genomes," Nucleic Acids Research, vol. 29, no. 1, pp. 22-28, 2001.

[39] K. Korenblat, Z. Volkovich, and A. Bolshoy, "Robustness of the whole-genome prokaryotic clustering based on gene lengths," Computational Biology and Chemistry, vol. 40, pp. 20-29, 2012.

[40] I. Borg and P. J. F. Groenen, Modern Multidimensional Scaling, Theory and Applications, Springer, New York, NY, USA, 2005.

[41] D. E. Knuth, Art of Computer Programming, Addison-Wesley, New York, NY, USA, 1973.

[42] P. Groenen, The majorization approach to multidimensional scaling: some problems and extensions [Ph.D. thesis], University of Leiden, 1993.

[43] J. G. Kemeny, "Mathematics without numbers," Daedalus, vol. 88, pp. 571-591, 1959.

[44] J. G. Kemeny and J. L. Snell, Mathematical Models in the Social Sciences, The MIT Press, Cambridge, UK, 1972.

[45] I. Bartholdi, C. A. Tovey, and M. A. Trick, "Voting schemes for which it can be difficult to tell who won the election," Social Choice and Welfare, vol. 6, no. 2, pp. 157-165, 1989.

[46] C. Dwork, R. Kumar, M. Naor, and D. Sivakumar, "Rank aggregation methods for the web," in Proceedings of the 10th International Conference on World Wide Web (WWW'01), pp. 613-622, 2011. 

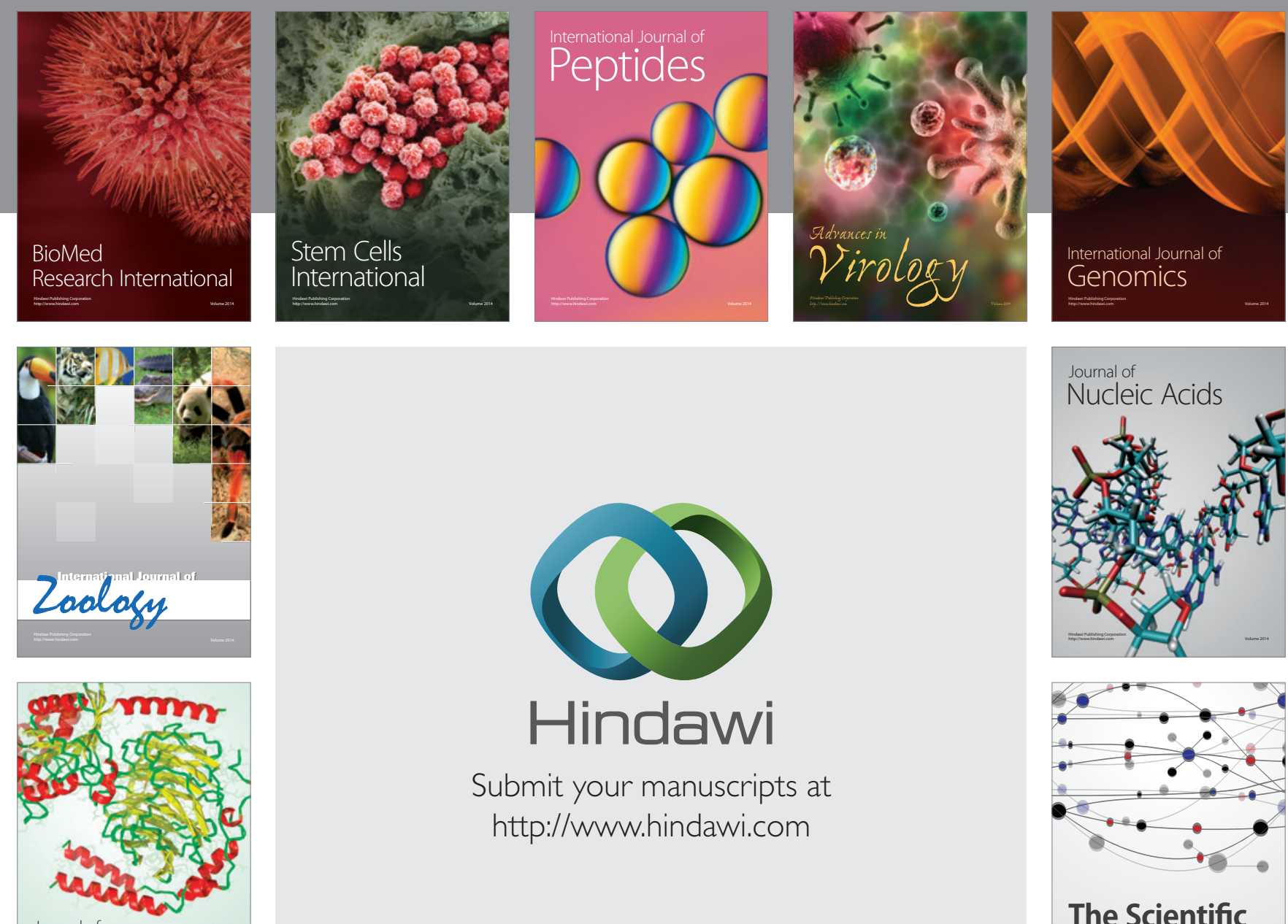

Submit your manuscripts at

http://www.hindawi.com

Journal of
Signal Transduction
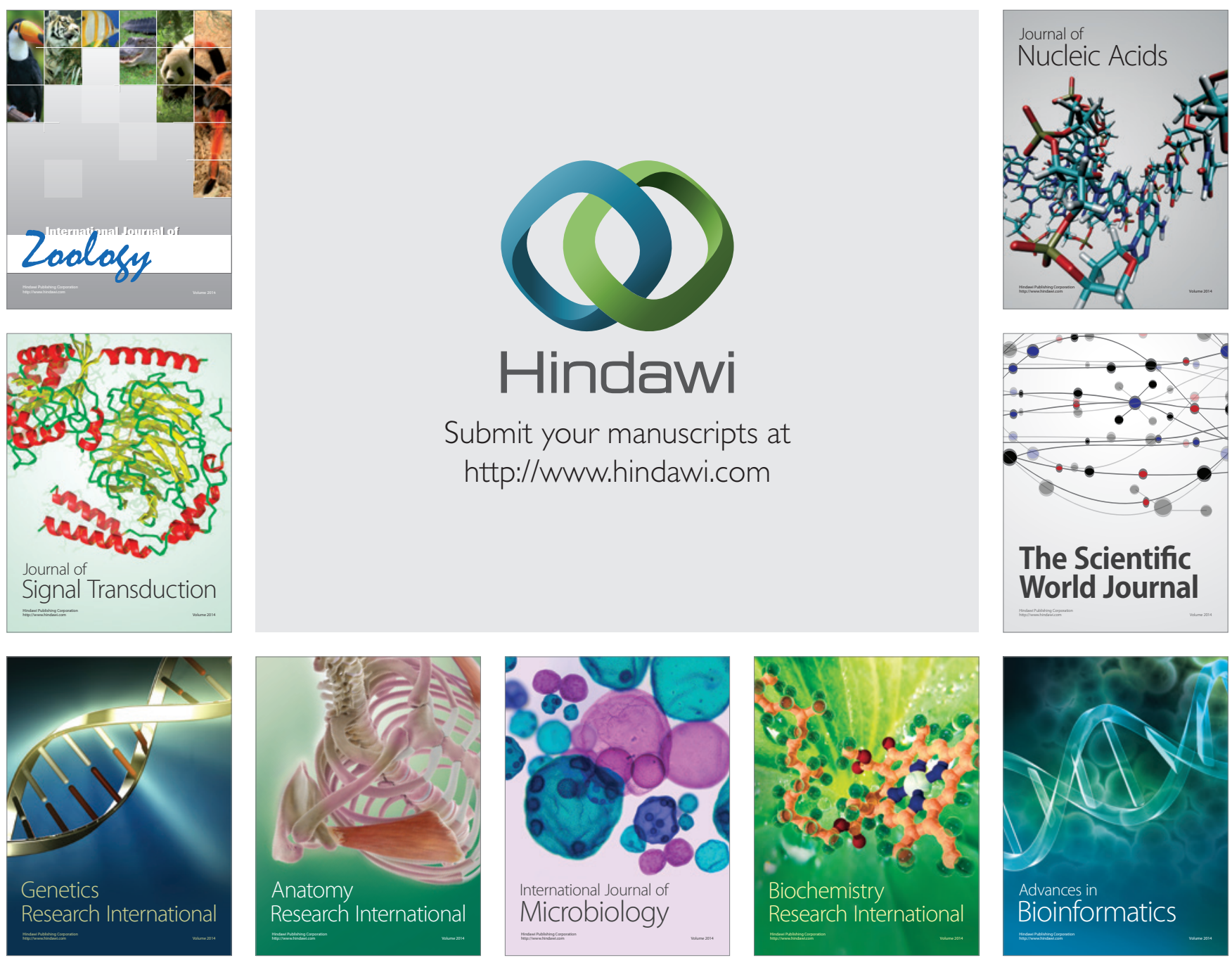

The Scientific World Journal
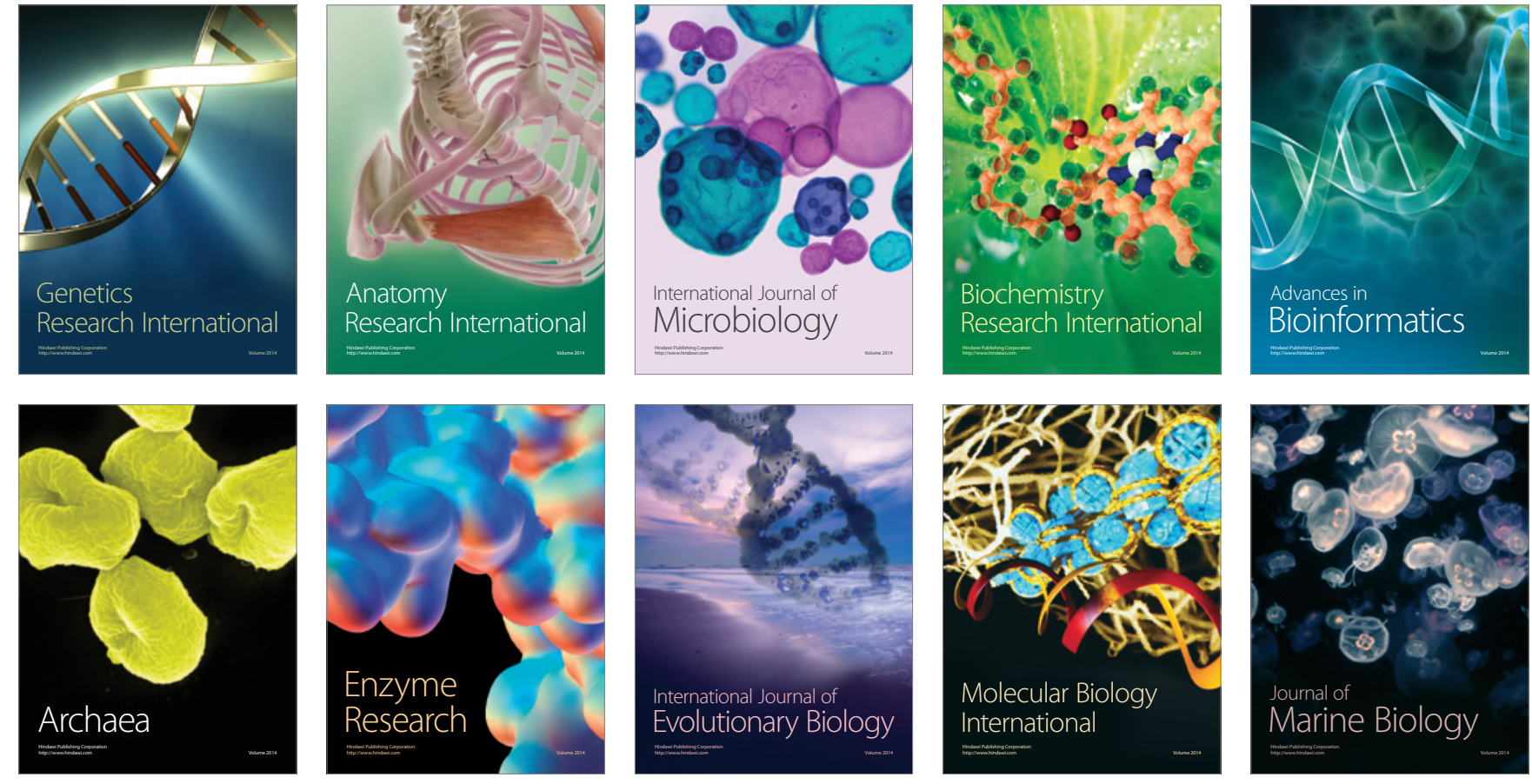